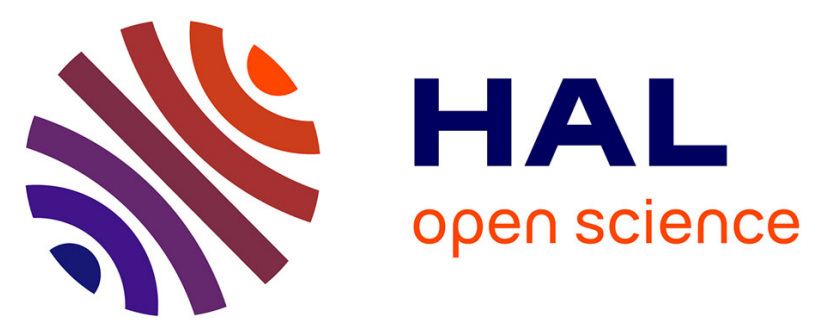

\title{
A method for MRI guidance of intercostal high intensity focused ultrasound ablation in the liver
}

Bruno Quesson, Mathilde Merle, Max Köhler, Charles Mougenot, Sebastien Roujol, Baudouin Denis de Senneville, Chrit Moonen

\section{- To cite this version:}

Bruno Quesson, Mathilde Merle, Max Köhler, Charles Mougenot, Sebastien Roujol, et al.. A method for MRI guidance of intercostal high intensity focused ultrasound ablation in the liver. Medical Physics, 2010, 37 (6), pp.2533-2540. 10.1118/1.3413996 . hal-01503914

\section{HAL Id: hal-01503914 \\ https://hal.science/hal-01503914}

Submitted on 29 Aug 2017

HAL is a multi-disciplinary open access archive for the deposit and dissemination of scientific research documents, whether they are published or not. The documents may come from teaching and research institutions in France or abroad, or from public or private research centers.
L'archive ouverte pluridisciplinaire $\mathbf{H A L}$, est destinée au dépôt et à la diffusion de documents scientifiques de niveau recherche, publiés ou non, émanant des établissements d'enseignement et de recherche français ou étrangers, des laboratoires publics ou privés. 


\section{A Method for MRI Guidance of Intercostal High Intensity Focused Ultrasound Ablation in the}

Liver

Bruno Quesson, Mathilde Merle, Max O Köhler, Charles Mougenot, Sebastien Roujol, Baudouin Denis

5 de Senneville, Chrit T Moonen

Running title

MR guided intercostal HIFU in the liver

10 Keywords

HIFU, liver, Rib, MR thermometry 


\begin{abstract}
High Intensity Focused Ultrasound (HIFU) is a promising method for the non-invasive treatment of liver tumors. However, the presence of ribs in the HIFU beam path remains problematic since it may lead to adverse effects (skin burns) by absorption and reflection of the incident beam at or near the bone surface. This paper presents a method based on Magnetic Resonance (MR) Imaging for identification of the ribs in the HIFU beam, and for selection of the transducer elements to deactivate. The ribs are visualized on anatomical images acquired prior to heating and manually segmented. The resulting regions of interest surrounding the ribs are projected onto the transducer surface by ray

20 tracing from the focal point. The transducer elements in the "shadow" of the ribs are then deactivated. The method was validated ex vivo and in vivo in pig liver during breathing under multi-slice real-time MR thermometry, using the proton resonance frequency shift method. Ex vivo and in vivo temperature data showed that the temperature increase near the ribs was important when HIFU sonications were performed with all elements active, whereas the temperature was reduced with deactivation of the

25 transducer elements located in front of the ribs. The temperature at the focal point was similar with and without deactivation of the transducer elements, indicative of the absence of heat efficacy loss with the proposed technique. This method is simple, rapid and reliable and enables intercostal HIFU ablation while sparing ribs and their surrounding tissues.
\end{abstract}




\section{Introduction}

Non invasive high-intensity focused ultrasound (HIFU) ablation of liver tumors has been demonstrated to be feasible in a clinical environment ${ }^{1-4}$. However, collateral damage such as skin burns ${ }^{1}$ has been reported because of poor acoustic coupling or the presence of ribs in the beam path.

35 This adverse effect is related to large differences in the acoustic impedance of soft tissue and bones ${ }^{5}$, resulting in heating of the bones ${ }^{6}$ by absorption of the acoustic energy and reflection of the incident beam toward the transducer. To overcome this limitation, it has been proposed to perform a partial rib resection prior to HIFU ablation of hepatocellular carcinoma ${ }^{7}$, but this procedure severely reduces the non invasiveness of the HIFU therapy.

Several methods have been proposed to perform intercostal sonications while reducing the acoustic illumination of the ribs. Botros et al. performed simulations to determine the excitation function of the HIFU transducer satisfying this condition ${ }^{8,9}$. Civale ${ }^{10}$ used a linearly segmented transducer and reported the results from acoustic field simulations and measurements on ex vivo ribs. Switching off one or several segments of the transducer resulted in a reduced temperature increase at 45 the bones. Liu et $a l^{11}$ performed a detailed 3D numerical analysis to investigate the feasibility of transrib focusing. They exploited CT images from patients to provide realistic modeling of the focused ultrasound propagation in the liver through the rib cage. They compared the simulated acoustic pressure, specific absorption rate of energy and temperature increase for a HIFU transducer with uniform excitation and with partial deactivation of a number of elements. Reduction of the energy deposition at the bones was obtained by deactivating the transducer elements for which the vector normal to the surface of the element was intercepted one of the ribs.

A more sophisticated approach is the time-reversal focusing ${ }^{12}$, based on the concept of the timereversal mirror introduced by Thomas and Fink ${ }^{13}$, where the phase aberration induced by ultrasound propagation through a heterogeneous medium can be compensated to retrieve the optimal acoustic 
intensity at the focal point. The method relies on an initial experiment in which the ultrasound wave front emitted from the focal point by an iserted probe is measured by the HIFU transducer elements. The phase aberrations introduced by the medium can then be compensated during HIFU sonication by generating the acoustic fields on each element with the opposite phase. Ex vivo transcostal HIFU based on this technique was presented by Aubry et $a l^{14}$, who performed a comparative analysis of the pressure

60 field distribution with all the elements active and with the time reversal technique. The quality of the focusing was improved by the latter technique due to compensation of diffraction effects, but only a slight increase of $4 \%$ in the measured pressure field was reported under these well controlled conditions. With this technique, the contribution of the ultrasound fields generated by the elements located in front of the bones to the total acoustic field can be considered negligible since only the relative phase of the electrical signals applied by each element are compensated, without compensation of the amplitude. More recently, an adaptive optimization method ${ }^{15}$ based on the analysis of the backscattered signals toward the HIFU transducer was proposed to identify the elements to activate, in order to minimize the reflected HIFU beam without requiring emission from the focal point with an invasive probe. However, this method was only validated on ribs immersed in a water tank and

70 selection of the elements from the backscattered ultrasound beam may be more difficult to perform in vivo in presence of a more complex tissue composition. Moreover, this method requires a precalibration step during which each transducer element sequentially emits a single pulse whose echo is analyzed by all elements to define the final excitation pattern. All ultrasound based methods require transmit/receive HIFU technology which is not available in current clinical devices.

From these different studies, it appears that the selective deactivation of transducer elements located in front of the ribs, despite being suboptimal as compared to the time reversal technique, provides a practical strategy for intercostal HIFU in the liver with an acceptable loss in heating efficiency at the focal point. The monitoring of the local temperature changes during intercostal liver 
HIFU ablation should ideally provide quantitative information about the local temperature distribution

80 around the focal point and near the bones. Magnetic Resonance (MR) temperature imaging with the Proton Resonance Frequency (PRF) $)^{16-18}$ method can provide such information in soft tissues. Kopelman et al have already reported MRgHIFU results obtained in the liver of healthy pigs ${ }^{19}$ and in a $\operatorname{dog}$ mode $^{20}$ under apnea. Daum et $a l^{21}$ used MR-thermometry to demonstrate that the temperature increase near the skin was important for transcostal HIFU sonications performed in pig cadavers.

85 However, no practical solution was proposed to overcome this problem. Respiratory motion can alter the precision of the thermometry in the liver due to motion related susceptibility changes. Correction strategies have been proposed ${ }^{22}$ to compensate for this effect allowing for real-time MR thermometry in mobile organs.

This paper presents a method for selection of the transducer elements to deactivate based on the

90 relative location of the focal point and the ribs as identified from anatomical MR images. The local temperature changes at the focal point and near the ribs were monitored in real-time during HIFU sonications performed ex vivo and in vivo in pig liver during breathing, with and without deactivation of the transducer elements located in front of the ribs. 


\section{Material and Methods}

\section{Ex vivo sample preparation}

The ex vivo samples consisted of a portion of thoracic cage of a pig, including ribs and muscle,

100 on top of which a piece of a pig liver was positioned. Two solid gel pads were put under the ribs and on top of the liver to ensure continuous ultrasound wave propagation in the near field and the far field of the HIFU beam path. The tissues used in these experiments were not degassed. A thin layer of a liquid mixture of water and ultrasound gel was inserted between the different parts of the sample to ensure continuous ultrasound coupling.

\section{Animal preparation for in vivo experiments}

In vivo experiments were performed on three pigs weighing approximately $50 \mathrm{~kg}$ and under general anesthesia, following the protocol described $\mathrm{in}^{23}$. The animals were sedated by intramuscular injection of $0.1 \mathrm{mg} / \mathrm{kg}$ of acepromazine (calmivet) (Vetoquinol, Lure, France) and anesthetized by IV

110 injection of $0.1 \mathrm{~mL} / \mathrm{kg}$ of pentobarbital (CEVA santé animale, Libourne, France). The anesthesia and analgesia were maintained with continuous IV injection of pentobarbital at a rate of $6 \mathrm{mg} / \mathrm{kg} / \mathrm{h}$ and ketamine (Imalgene 1000, Merial, Lyon, France) at a rate of $7.2 \mathrm{mg} / \mathrm{kg} / \mathrm{h}$ through two different ear veins. The right side of the abdomen was shaved and depilatory cream was applied in order to remove the hairs and ensure good acoustic coupling. The animals were positioned right decubitus on the MR

115 compatible HIFU platform (see below for details), and maintained under assisted ventilation (respirator paraPac, ResMed SA, France) during the procedure, using a volume of $400 \mathrm{~mL}(45 \%$ O2) at a frequency of $20 \mathrm{bpm}$. The cardiac frequency and the rectal temperature were monitored during the procedure. After completion of the MRgHIFU experiments the animals were euthanized by intravenous injection of Dolethal (Vetoquinol, Lure, France). The experimental protocol was approved by the 
120 ethical committee of the institution (agreement \#AP02092009).

\section{MR compatible HIFU platform}

The thermal ablation experiments were performed with a MR compatible HIFU platform (Philips Healthcare, Finland) designed for the treatment of uterine fibroid, integrated into the 1.5T

125 Achieva-Intera MRI (Philips Healthcare, The Netherlands). The HIFU transducer (256 elements, $12 \mathrm{~cm}$ focal length and $13 \mathrm{~cm}$ aperture) can be mechanically positioned inside a degassed water tank and the voltage amplitude and phase applied to each element can be adjusted independently. Acoustic propagation was achieve through a thin circular membrane located on top of the platform. MR images were acquired with the receiver coil integrated into the HIFU platform.

MR imaging and data processing for transducer positioning and selection of the elements to deactivate

A 3D proton-density weighted fast gradient echo sequence was acquired prior to sonications, with an echo time (TE) of $4.6 \mathrm{~ms}$, a repetition time (TR) of $8.57 \mathrm{~ms}$, a $15^{\circ}$ water selective binomial

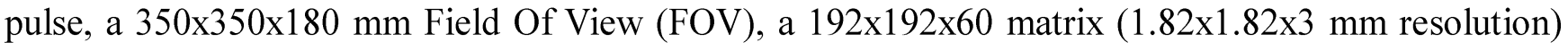

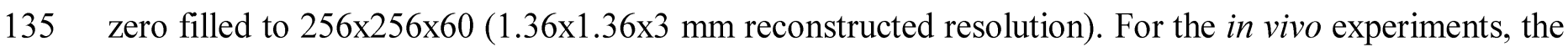
sequence was triggered on the respiratory signal, leading to a $2 \min 16 \mathrm{~s}$ acquisition duration. The reconstructed volume was transferred (Figure 1) to a home made software written in IDL (RSI, ITT, Visual Information Solutions) which allowed for visualization of the images in the transverse, sagittal and coronal orientations. The $\mathrm{x}, \mathrm{y}$ and $\mathrm{z}$ coordinates of the transducer position were imported to allow

140 for superimposition of the propagation cone on the displayed images. After selection of the desired sonication location in the liver, the actual transducer position was updated on the HIFU control console. Horizontal slices located in the HIFU near field were visualized sequentially to identify the images containing the bones (Figure 1b). For each selected slice, a region of interest (ROI) surrounding each 
bone was manually drawn (Figure 1c). Then, each ROI was projected onto the transducer surface by

145 oblique ray tracing from the selected focal point (see Figure 1d) and each point included in the ROIs.

For this purpose, a mask corresponding to the active surface of a virtual flat transducer array was constructed. The projection of each transducer element apparent surface viewed from the focal point was performed in a horizontal plane located at the top part $(20 \mathrm{~mm})$ of the transducer $(1.6 \mathrm{~s}$ processing time for a $140 \mathrm{~mm}$ square FOV and a $204 \times 204$ pixels resolution) after importing the

150 coordinates and size of each transducer element. The analytical equations of the lines crossing the focal point and each of the 15372 pixels included into the active surface were iteratively computed. For each line, the pixel of the virtual transducer was considered to be in the shadow of the rib if the line intercepted the user defined ROI surrounding the ribs (Figure 1e). The transducer elements whose surface was covered by at least $50 \%$ of this shadow were included in the list of elements to deactivate.

155 For each of the elements to be deactivated, the electrical output of the corresponding channel on the HIFU generator was turned off by including specific command lines in the header of file describing the sonication characteristics (acoustic power, sonication location and duration) executed on the HIFU command console. The computation time for the whole process was less than $10 \mathrm{~s}$.

\section{MR thermometry acquisition and processing}

MR thermometry was performed before, during, and after HIFU sonication with the Proton Resonance Frequency shift method. For ex vivo experiments, the acquisition sequence was a gradient recalled segmented Echo-Planar Imaging (EPI) with 5 k-space lines acquired per repetition time (TR), with and effective echo time of $15 \mathrm{~ms}$, a TR of $66 \mathrm{~ms}$, a $20^{\circ}$ water selective binomial pulse to avoid the

165 signals of the lipids, a $208 \times 260 \mathrm{~mm}$ FOV, a 100x128 Matrix (2.08×2.03 mm in plane resolution) zero filled to $128 \times 128$ (reconstructed in plane resolution of $2.03 \times 2.03 \mathrm{~mm}$ ) and a $6 \mathrm{~mm}$ slice thickness. Two slices were acquired each $1.3 \mathrm{~s}$, the first one in the vertical orientation for visualization of the 
temperature in the propagation cone of the HIFU and the second one in the horizontal plane below the ribs for visualization of the temperature evolution in the near field of the ultrasound beam.

170 For in vivo experiments, a similar approach was used but with a faster acquisition sequence to reduce intra-scan motion associated with the breathing. For this purpose, a gradient recalled single shot EPI was performed with $59 \mathrm{k}$-space lines acquired per TR, an effective echo time of $23 \mathrm{~ms}$, a TR of 200 ms, a water selective binomial pulse with a flip angle of $60^{\circ}$, a 160x300 mm FOV, and 59x112 matrix (2.72x2.68 $\mathrm{mm}$ in plane resolution) zero filled to $128 \times 128$ (reconstructed in plane resolution of $1752.34 \times 2.34 \mathrm{~mm}$ ) and a $6 \mathrm{~mm}$ slice thickness. Two slices were acquired in the horizontal plane, one located at the focal point and one near the ribs. A third slice was positioned perpendicular to the two other slices to visualize the propagation of the HIFU beam in the liver. For each slice, the readout direction was aligned with the superior/inferior direction. Two saturation slabs were also added to avoid fold over artifacts. The three temperature images were continuously updated each $600 \mathrm{~ms}$.

Magnitude and phase images were reconstructed by the MRI system and streamed in real time to a separate workstation (dual processor Intel 3.1GHz Penryn, four cores, 8GB RAM). Temperature images were calculated and displayed on the fly from in house developed software ("RealTI") written in $\mathrm{C}++$ and IDL (ITT, Visual Information Solutions). A small ROI of 5x5 pixels was selected outside the heated region on each slice to correct for the temporal field drift. For the in vivo experiments,

185 magnitude and phase images were processed to compensate for motion artifacts in the temperature maps with the multi-baseline correction technique ( $\mathrm{se}^{22}$ for details). The maximal temperature increase at the focal spot was reported. A square ROI of 40x40 pixels was positioned around the heated area in the MR temperature slice located in the near field. The mean temperature increase over a $100 \mathrm{~s}$ postsonication period starting $30 \mathrm{~s}$ after the end of the sonication was computed for each pixel included in

190 the selected ROI. The number of pixels having reached a mean temperature increase of $3^{\circ} \mathrm{C}, 5^{\circ} \mathrm{C}, 7^{\circ} \mathrm{C}$ and $10^{\circ} \mathrm{C}$ was reported for each experimental condition. The regions showing hypointense signal in the 
magnitude images were masked out on the temperature images and excluded from the ROI since the precision of the phase estimate, and therefore the subsequent temperature estimate, is inversely proportional to the signal-to-noise ratio (SNR) of the magnitude images ${ }^{24}$..

\section{Results}

Figure 2 compares the results obtained on ex vivo tissue samples. Two sonications were performed at the same location at $5 \mathrm{~cm}$ depth in the liver, without (left) and with (right) deactivation of the elements located in front of the ribs. The ribs were seen as a hypo-intense region on the four slices and three ROIs were drawn (see Figure 1c) delineating the ribs. After projection of these ribs onto the transducer surface, 126 elements were identified as being located in the shadow of the ribs. The acoustic power delivered per element $(0.47$ Wacc, corresponding to a total acoustic power of $120 \mathrm{~W}$ when all elements were active and $60 \mathrm{~W}$ when deactivating $50 \%$ of the elements, respectively) was identical for both conditions. Moreover, in order to avoid potential changes in acoustic properties due

205 to temperature increases close to the bones in the near field, the experiment with all elements active was performed last. Figure $2 \mathrm{a}$ and $2 \mathrm{~b}$ show temperature images acquired at the end of the $30 \mathrm{~s}$ HIFU sonication. In the vertical slice (Figure 2a), a hot spot (black arrow) is observed at the focal point and in tissue surrounding the bones (see white arrow). In the horizontal slice located below the ribs (Figure 2b), a spatially inhomogeneous heating is observed in the soft tissue surrounding the ribs. In the 210 experiment performed with deactivation of the elements (right column in Figure 2), a similar temperature distribution is observed around the focal spot (Figure 2c) whereas the temperature increase near the bones is reduced (see images $\mathrm{c}$ and image d). The temperature images obtained after 120 seconds of cooling (second row of images) show a residual temperature elevation near the ribs (images e and f) and an absence of significant temperature increase when the elements were deactivated. The 215 analysis of the temporal evolution of the temperature at the focal point (Figure 2i) for both conditions 
show very similar curves with maximal temperature increases of $7^{\circ} \mathrm{C}$, indicating an absence of significant acoustic intensity loss with the proposed method. In contrast, the temperature evolution in a single pixel (white arrow in Figure 2a) located near a rib shows a substantial difference (Figure $2 \mathrm{j}$ ). The maximal near-field temperature increase reached at the end of the sonication was $15^{\circ} \mathrm{C}$ when all the 220 elements were used whereas it remained below $4^{\circ} \mathrm{C}$ when the selected elements were deactivated. A slow temperature decrease to $9^{\circ} \mathrm{C}$ was observed after 2 minutes of cooling in the experiment performed with all the elements active, higher than the maximal temperature observed at the focal point.

Figure 3 shows anatomical images obtained in vivo of a pig under ventilation. In this example, the selected sonication point was located $4.5 \mathrm{~cm}$ inside the liver (see Figures $3 \mathrm{a}$ and $3 \mathrm{~b}$ ). Image analisys 225 identified two ribs within the HIFU beam (Figure 3c) and the projection of the ROI onto the transducer surface resulted in deactivation of 124 elements (Figure 3d). Two sonications were performed consecutively at the same location, separated by a cooling delay of 20 minutes. The first sonication was performed with deactivation of the elements located in front of the ribs and the second experiment was performed with all the elements active. For both experiments, 1 acoustic watt (Wac) per transducer 230 element was applied during $20 \mathrm{~s}$.

Figure 4 compares the temperature data when all elements are active and when the selected elements are deactivated. Similar distributions of the temperature were observed on temperature images acquired at the end of HIFU deposition in the sagittal slice containing the HIFU beam path (Figures 4a and $4 \mathrm{~b}$ ), with a focal point heating inside the liver and residual heating in the subcutaneous tissues. The 235 temperature evolution at the focal point is shown in Figure 4c. A similar temperature increase of $17^{\circ} \mathrm{C}$ is observed for both experimental conditions, indicating an absence of acoustic loss at the focal point when the elements are deactivated. After application of HIFU, the temperature rapidly returned toward its initial value due to perfusion and heat diffusion, with nearly identical evolution for both experimental conditions. Note that after 30 seconds of cooling (time $=100 \mathrm{~s}$ on Figure $4 \mathrm{c}$ ), the residual 
240 temperature increase was $4^{\circ} \mathrm{C}$. The temperature data in the orthogonal horizontal slice located at the focal spot (data not shown) also show similar results for both conditions.

Figure 5 displays the results of the temperature evolution near the ribs for the same experiment. The comparative analysis of the temperature evolution in a single pixel (Figure 5a) shows a reduction by a factor of $2(56 \%$ reduction $)$ in the temperature increase when the elements were deactivated $\left(6.2^{\circ} \mathrm{C}\right.$ 245 average temperature increase), as compared to the experiment performed with all elements active $\left(14.3^{\circ} \mathrm{C}\right.$ average temperature increase). Note that the temperature evolution is different with respect to that at the focal point, since the temperature reaches a plateau 30 seconds after the end of the sonication and remains nearly constant over the observation period ( $2 \min 30 \mathrm{~s}$ post-sonication). A reduction of $70 \%$ of the mean temperature increase was found in a single pixel located in the cartilage (Figure $5 \mathrm{~b}$ ), with a mean temperature increase of $7.2^{\circ} \mathrm{C}$ when all the elements were active and $2.1^{\circ} \mathrm{C}$ when the 124 elements were deactivated, respectively. The average temperature maps (Figures $5 \mathrm{c}$ and $5 \mathrm{~d}$ ) calculated over 100 seconds during the cooling period showed, as expected, that the temperature increase was higher in the vicinity of the ribs when no elements were deactivated (Figure 5c), whereas the temperature increase was lower and more spatially homogeneous when the 124 elements were 255 deactivated.

Table 1 presents the in vivo results obtained on each animal, with and without deactivation of the transducer elements located in front of the ribs. For the three animals, approximately half of the elements were deactivated. The temperature increase at the focal point ranged between $15^{\circ} \mathrm{C}$ and $17^{\circ} \mathrm{C}$ with or without deactivation of the elements. The maximal temperature increase in the selected ROI in 260 near field was below $10^{\circ} \mathrm{C}$ for the three experiments performed with partial deactivation of the transducer elements, whereas it reached $15^{\circ} \mathrm{C}$ and $19^{\circ} \mathrm{C}$ on the first and third animals, respectively. On the second animal, the sonication performed with all the elements active resulted in an emergency stop of the sonication after 10 seconds due to overheating of the transducer itself. This overheating was 
attributed to the reflection of the acoustic beam from the ribs toward the transducer surface, onto a

265 thermal sensor embedded in the transducer active surface. The number of pixels having reached the different average temperature threshold values $\left(3^{\circ} \mathrm{C}, 5^{\circ} \mathrm{C}, 7^{\circ} \mathrm{C}\right.$ and $\left.10^{\circ} \mathrm{C}\right)$ in the near field was systematically lower with deactivation of the transducer elements. No pixels reached a $10^{\circ} \mathrm{C}$ temperature increase under these conditions, whereas more than 20 pixels displayed an average temperature increase higher or equal to this threshold for animals 1 and 3.

\section{Discussion}

This paper presents a simple and practical solution based on anatomical MR images for selection of the transducer elements to deactivate in order to reduce the deposition of acoustic energy at

275 the ribs. The method proposed in the present work is based on the identification of the obstacles within the beam path from MR images and their projection onto the transducer surface with an oblique ray tracing from the HIFU target. The acquisition sequence allowed for a clear visualization of the bones, cartilages and soft tissues. In principle, this method may be used with any MR sequence, with the condition that sufficient contrast between the obstacle and the surrounding tissues exists. Therefore, it

280 could be applied for identification of any problematic structure (bowels, scars, bladder, etc...) taking advantage of the excellent image quality and contrast provided by MRI. Such an approach may be more difficult with ultrasound based imaging because the presence of these reflecting media complicates the visualization of both the obstacles and the targeted point located behind these obstacles. The small increase in duration related to the acquisition and processing of the 3D anatomical 285 images will not impair the overall therapeutic procedure.

The proposed algorithm allows deactivation of the elements of the transducer based on a geometric projection of the shadow of the ribs. This simple method was suggested by Liu $e t a l^{11}$ in their simulations. The method was here applied in order to provide a proof of concept of the method based 
on the analysis of anatomical MRI data and to evaluate its effect on actual temperature distribution both

290 ex vivo and in vivo, with the help of real-time MR thermometry. Alternatively, the MR images could be exploited in a more sophisticated manner to provide input data to already proposed algorithms for optimization of energy deposition $8,12,15,25$. However, since the temperature evolutions at the target location was found nearly identical with or without deactivation of the elements in front of the ribs in both ex vivo and in vivo experiments, no substantial loss in acoustic intensity at the target location is 295 apparent with this simple method. This is in good agreement with the ex vivo results reported by Aubry et al (see table 2 in $^{14}$ ) who measured a $4 \%$ difference in acoustic intensity at the focal point when all the elements were active as compared to the theoretically optimal solution offered by the time reversal method.

In the present work, the transducer elements were deactivated when the surface covered by the 300 shadow of the bones exceeded $50 \%$ of the element surface. Despite the fact that the resulting temperature evolution at the focal point was equivalent with and without deactivation of the elements, there is no proof that this choice is optimal for minimizing the acoustic field produced at the bones. However, setting more restrictive conditions, such as for example excluding any element even partially covered by the shadow, would have resulted in 12 and 10 additional elements to deactivate for the ex 305 vivo and in vivo experiments, respectively. Since these elements were located all around the border of the shadow, the resulting acoustic energy was spread in different locations on the ribs which did not result in a important increase of the acoustic field illuminating the bones. More sophisticated approaches could be proposed, such as modulation of the power delivered to these elements as a function of the partial coverage and/or phase compensation of the diffracting obstacles (similar to the 310 time reversal technique), but this was out of the scope of the present work.

One of the limiting factors of the proposed method is that the deactivated elements of the HIFU transducer depend on the ROIs drawn by the user, who may either exclude very large regions and 
decrease the efficacy of the acoustic emission or include too many problematic structures and increase the risk of creating collateral damage. To overcome this limitation, semi-automatic or automatic 315 segmentation techniques could be implemented. .

The closest focalization distance behind the center of the obstacle is restricted by the relative dimension of the obstacles in the direction orthogonal to the propagation axis and the transducer aperture and focal length. Taking into account the transducer characteristics used in this study and a typical bone dimension of $13 \mathrm{~mm}$ in vivo as measured on 3D anatomical images, the minimal distance of focalization behind the center of the bone leading to total deactivation of the transducer surface was $10 \mathrm{~mm}$. Another important aspect concerns the modification of the spatial distribution of the acoustic intensity in case of partial activation of the transducer emitting surface, with possible increase of the the focal spot length and appearance of side lobes. As a consequence, intecostal HIFU ablation may require to take into account sonication location, size of the obstacle, transducer characteristics, resulting acoustic intensity pattern and tissue thermal parameters (specific heat, thermal diffusivity and perfusion).

The online monitoring of the temperature evolution in multiple orientations and locations offer the advantage of providing a direct feedback to the physician. Therefore, this information may be included in automatic control algorithms ${ }^{26}$ in order to increase the patient safety in clinical practice.

330 Our results provide a quantitative estimate of the influence of tissue overheating around the ribs when sonications are performed without any precaution. Despite the fact that thermometry based on the PRF technique does not provide quantitative information of the actual temperature changes inside the bones, the important and localized heating in the surrounding soft tissues can be attributed to rib heating. The observed delayed heating after the end of the sonication can be related to thermal diffusion from the

335 bones to the adjacent soft tissues. For ex vivo tissue samples, the maximal temperature increase was higher near the bone than at the focal point and slowly decreased after the end of the sonication. This is 
in good agreement with the important absorption of acoustic energy by the bones as compared to soft tissue at the used ultrasound frequency ${ }^{5,11,27}$. For the in vivo experiments, the maximal temperature rise was similar $\left(15^{\circ} \mathrm{C}\right)$ near the rib and at the focal point when all the elements were active, but the

340 temperature increase near the ribs was reduced by deactivation of the elements. However, for both ex vivo and in vivo conditions, the near-field temperature reached a plateau and no temperature decrease was observed during the 2 minutes 30 seconds of cooling time. In contrast to the near field, the temperature at the focal point returned rapidly toward its initial value due to tissue perfusion and rapid thermal diffusion caused by strong temperature gradients. The lack of temperature decrease near the

345 ribs may be attributed to the lower perfusion as compared to the liver and to thermal insulation provided by the fat layer in the subcutaneous tissues. Our results are in good agreement with the MR temperature data presented by Daum et al. ${ }^{21}$ for sonications performed on cadavers of pigs with all the elements active, who also showed slow temperature evolution after the end of the sonication. In our in vivo results, the accumulated thermal $\operatorname{dose}^{28,29}$, assuming a homogeneous baseline temperature of $35036.5^{\circ} \mathrm{C}$ (taken from the rectal temperature), reached the threshold of 240 equivalent minutes at $43^{\circ} \mathrm{C}$ in the soft tissue surrounding the ribs, when all elements are activated. This thermal dose threshold has previously been reported to be a relevant indicator of the induced thermal lesions ${ }^{30,31}$. The analysis of the slice located near the ribs did not reveal motion associated with the respiratory activity. In case of motion of the ribs during sonication, the list of the transducer elements to deactivate could be 355 dynamically updated based on analysis of the magnitude images acquired with the thermometry sequence. Alternatively, the combined footprint of the ribs over the complete respiratory cycle could also be analyzed prior to sonication to deactivate the required elements, at the cost of a reduced acoustic intensity at the focal point.

In conclusion, this paper presents a method for selecting the transducer elements to deactivate 360 for a given patient position in order to avoid unnecessary deposition of acoustic energy on the ribs, by 
taking into account the HIFU transducer characteristics (focal length, location of each element) and sonication location. Our results confirm that deactivation of the elements in front of the ribs is mandatory to reduce the risks of excessive near-field heating and skin burns. The proposed method can therefore increase the patient safety by offering a fully controlled HIFU ablation of the liver while 365 protecting the ribs and surrounding healthy tissues, by combining MR compatible multi-elements transducer technology, MR anatomical imaging and real-time MR thermometry.

\section{Acknowledgments}

Audrey Osowsky and Jean-Patrick Chenu are greatly appreciated for animal care and Matti Lindström and Matti Tillander are appreciated for their technical help in HIFU hardware handling. This project was funded by the Agence National de la Recherche (project "MRgHIFU-ALKT"), the Foundation InNaBioSanté (project "ULTRAFITT", la Ligue National contre le Cancer, and by the Conseil Régional d'Aquitaine. 
Table 1: Summary of the results of the three in vivo experiments performed with and without deactivation of the transducer elements located in front of the ribs. For each condition, $1 \mathrm{Wac} / \mathrm{element}$ was emitted during $20 \mathrm{~s}$. The reported temperature are rounded to the closest integer values. The maximal temperature increase at the focal point and in the near field ROI are reported in the third and fourth columns, respectively. The last four columns indicate the number of pixels in this ROI having reached an average temperature increase higher or equal to $3^{\circ} \mathrm{C}, 5^{\circ} \mathrm{C}, 7^{\circ} \mathrm{C}$ and $10^{\circ} \mathrm{C}$, respectively. $*$ : the maximal temperature increase is not reported since the sonication 385 automatically halted after 10 s sonication due to internal security check of the HIFU system (see text for details).

\begin{tabular}{|c|c|c|c|c|c|c|c|}
\hline \multirow[b]{3}{*}{ Animal \# } & \multirow[b]{3}{*}{ \# of elements } & \multirow{3}{*}{$\begin{array}{l}\text { Focal point } \\
\mathrm{T} \max \left[{ }^{\circ} \mathrm{C}\right]\end{array}$} & \multicolumn{5}{|c|}{ Near field } \\
\hline & & & \multirow[b]{2}{*}{$\mathrm{T} \max \left[{ }^{\circ} \mathrm{C}\right]$} & \multicolumn{4}{|c|}{ \# of pixels with $\mathrm{T}$ higher than } \\
\hline & & & & $3^{\circ} \mathrm{C}$ & $5^{\circ} \mathrm{C}$ & $7^{\circ} \mathrm{C}$ & $10^{\circ} \mathrm{C}$ \\
\hline \multirow[t]{2}{*}{1} & 132 & 17 & 7 & 115 & 41 & 0 & 0 \\
\hline & 256 & 16 & 15 & 183 & 111 & 61 & 21 \\
\hline \multirow[t]{2}{*}{2} & 134 & 16 & 8 & 120 & 43 & 5 & 0 \\
\hline & 256 & $\mathrm{na}^{*}$ & - & - & - & - & - \\
\hline \multirow[t]{2}{*}{3} & 126 & 15 & 7 & 88 & 19 & 0 & 0 \\
\hline & 256 & 15 & 19 & 167 & 123 & 80 & 22 \\
\hline
\end{tabular}




\section{Figure captions}

Figure 1 : Schematic diagram (a) of the proposed method for selecting the transducer elements to deactivate. Anatomical images are imported into the graphical user interface for : selection (b) of the target point (the red lines show the HIFU propagation cone on a transverse slice and the white dashed line shows the horizontal slice displayed in c), manual segmentation (c) of the bones (yellow

395 ROIs) within the beam path (red circle), projection (d) of the shadow (yellow) of the ROIs onto the transducer surface (white bars on the transducer surface) by ray tracing from the targeted point (white dashed lines), and visualization (e) of the shadow of the ROIs on the 256 transducer elements distributed on the transducer surface and determination of the elements to deactivate.

400 Figure 2 : MR temperature data obtained during HIFU sonications performed on ex vivo samples. Images (a-b) and (c-d) show color coded vertical and horizontal temperature images (temperature scale is indicated on top right of image (a)) superimposed on magnitude images obtained at the end of the HIFU sonication with all elements active $(\mathrm{a}, \mathrm{b})$ and after deactivation of the 126 elements (c, d). Images (e, f) and $(g, h)$ show temperature images acquired $120 \mathrm{sec}$ after the end of HIFU

405 sonication for both conditions. The graph in (i) compares the temperature evolution in the pixel located at the focal point (black arrow head in a) with all elements active (red) and after deactivation of the 126 elements (black). The graph in (j) compares the temperature evolution in a pixel located near a rib (white arrow head in image a) within the HIFU beam.

410 Figure 3 : Images showing the experimental configuration of the MRgHIFU experiments performed in vivo in the liver of a pig. Coronal (a), transverse (b) and sagittal (c) views of the animal positioned on top of the MRgHIFU platform. The red lines indicate the borders of the HIFU propagation cone and the white dashed lines indicate the position of the MR-thermometry slices. The yellow contours in (c) show the two ROIs surrounding the ribs and the image displayed in (d) 
415 shows the result of the projection of these ROIs onto the transducer surface.

Figure 4 : Comparison of temperature data during HIFU sonications performed in pig liver in vivo, without and with deactivation of the transducer elements. Images on the top show temperature images (color scale is provided on the right) in the sagittal orientation, with all the elements active (a) and after deactivation of the 124 elements (b). The graph displayed in (c) compares temperature evolution at the focal point (white arrow head on (a)) without (red curve) and with (black curve) deactivation of the elements. The HIFU sonication duration is represented by the gray rectangle.

Figure 5 : Comparison of temperature data near the ribs during HIFU sonications performed in pig 425 liver in vivo, without and with deactivation of the transducer elements. The graphs show the temperature evolution in a single pixel located (a) near the rib and (b) in the cartilage (see white points for exact location in each insert). The red curves show the temperature evolution when all the HIFU elements are active and the black curves show the temperature evolution when 124 elements were deactivated. The HIFU sonication duration $(20 \mathrm{~s})$ is represented by the gray rectangles. The 430 horizontal bars show the average values of temperature between 100 and 200 seconds. Images (c) and (d) are maps of the average temperature values (the color scale is indicated on the right) in each pixel between 100 and $200 \mathrm{~s}$ (vertical dashed blue lines in a and b). 


\section{References}

435 1. F. Wu, Z. B. Wang, W. Z. Chen, W. Wang, Y. Gui, M. Zhang, G. Zheng, Y. Zhou, G. Xu, M. Li, C. Zhang, H. Ye and R. Feng, "Extracorporeal high intensity focused ultrasound ablation in the treatment of 1038 patients with solid carcinomas in China: an overview," Ultrason Sonochem 11, 149-154 (2004).

2. F. Wu, Z. B. Wang, W. Z. Chen, H. Zhu, J. Bai, J. Z. Zou, K. Q. Li, C. B. Jin, F. L. Xie and H.

B. $\mathrm{Su}$, "Extracorporeal high intensity focused ultrasound ablation in the treatment of patients with large hepatocellular carcinoma," Ann Surg Oncol 11, 1061-1069 (2004).

3. R. O. Illing, J. E. Kennedy, F. Wu, G. R. ter Haar, A. S. Protheroe, P. J. Friend, F. V. Gleeson, D. W. Cranston, R. R. Phillips and M. R. Middleton, "The safety and feasibility of extracorporeal high-intensity focused ultrasound (HIFU) for the treatment of liver and kidney tumours in a Western population," Br J Cancer 93, 890-895 (2005).

4. J. E. Kennedy, F. Wu, G. R. ter Haar, F. V. Gleeson, R. R. Phillips, M. R. Middleton and D. Cranston, "High-intensity focused ultrasound for the treatment of liver tumours," Ultrasonics 42, 931-935 (2004).

5. S. A. Goss, L. A. Frizzell and F. Dunn, "Ultrasonic absorption and attenuation in mammalian tissues," Ultrasound Med Biol 5, 181-186 (1979).

6. H. L. Liu, N. McDannold and K. Hynynen, "Focal beam distortion and treatment planning in abdominal focused ultrasound surgery," Med Phys 32, 1270-1280 (2005).

7. H. Zhu, K. Zhou, L. Zhang, C. Jin, S. Peng, W. Yang, K. Li, H. Su, W. Chen, J. Bai, F. Wu and Z. Wang, "High intensity focused ultrasound (HIFU) therapy for local treatment of hepatocellular carcinoma: role of partial rib resection," Eur J Radiol 72, 160-166 (2009).

8. Y. Y. Botros, E. S. Ebbini and J. L. Volakis, "Two-step hybrid virtual array ray (VAR) technique for focusing through the rib cage," IEEE Trans Ultrason Ferroelectr Freq Control 45, 989-1000 (1998).

9. Y. Y. Botros, J. L. Volakis, P. VanBaren and E. S. Ebbini, "A hybrid computational model for 
ultrasound phased-array heating in presence of strongly scattering obstacles," IEEE Trans Biomed Eng 44, 1039-1050 (1997).

10. J. Civale, R. Clarke, I. Rivens and G. ter Haar, "The use of a segmented transducer for rib sparing in HIFU treatments," Ultrasound Med Biol 32, 1753-1761 (2006).

11. H. L. Liu, H. Chang, W. S. Chen, T. C. Shih, J. K. Hsiao and W. L. Lin, "Feasibility of transrib focused ultrasound thermal ablation for liver tumors using a spherically curved 2D array: a numerical study," Med Phys 34, 3436-3448 (2007).

12. M. Tanter, M. Pernot, J. F. Aubry, G. Montaldo, F. Marquet and M. Fink, "Compensating for bone interfaces and respiratory motion in high-intensity focused ultrasound," Int J Hyperthermia 23, 141-151 (2007).

470 13. J. L. Thomas, F. Wu and M. Fink, "Time reversal focusing applied to lithotripsy," Ultrason Imaging 18, 106-121 (1996).

14. J. F. Aubry, M. Pernot, F. Marquet, M. Tanter and M. Fink, "Transcostal high-intensity-focused ultrasound: ex vivo adaptive focusing feasibility study," Phys Med Biol 53, 2937-2951 (2008).

15. E. Cochard, C. Prada, J. F. Aubry and M. Fink, "Ultrasonic focusing through the ribs using the DORT method," Med Phys 36, 3495-3503 (2009).

16. V. Rieke, K. K. Vigen, G. Sommer, B. L. Daniel, J. M. Pauly and K. Butts, "Referenceless PRF shift thermometry," Magn Reson Med 51, 1223-1231 (2004).

17. V. Rieke and K. Butts Pauly, "MR thermometry," J Magn Reson Imaging 27, 376-390 (2008).

18. B. Quesson, J. A. de Zwart and C. T. Moonen, "Magnetic resonance temperature imaging for guidance of thermotherapy," J Magn Reson Imaging 12, 525-533 (2000).

19. D. Kopelman, Y. Inbar, A. Hanannel, D. Freundlich, D. Castel, A. Perel, A. Greenfeld, T. Salamon, M. Sareli, A. Valeanu and M. Papa, "Magnetic resonance-guided focused ultrasound surgery (MRgFUS): ablation of liver tissue in a porcine model," Eur J Radiol 59, 157-162 (2006). 
T. Salomon, M. Sareli, A. Valeanu and M. Papa, "Magnetic resonance-guided focused ultrasound surgery (MRgFUS). Four ablation treatments of a single canine hepatocellular adenoma," HPB (Oxford) 8, 292-298 (2006).

21. D. R. Daum, N. B. Smith, R. King and K. Hynynen, "In vivo demonstration of noninvasive thermal surgery of the liver and kidney using an ultrasonic phased array," Ultrasound Med Biol 25, 1087-1098 (1999).

22. B. D. de Senneville, C. Mougenot and C. T. Moonen, "Real-time adaptive methods for treatment of mobile organs by MRI-controlled high-intensity focused ultrasound," Magn Reson Med 57, 319-330 (2007).

23. C. Goldmann, A. Ghofrani, B. Hafemann, P. Fuchs, R. Khorram-Seffat, M. Afify, W. Kupper and N. Pallua, "Combination anesthesia with ketamine and pentobarbital: a long-term porcine model," Res Exp Med (Berl) 199, 35-50 (1999).

24. J. Sijbers, D. Poot, A. J. den Dekker and W. Pintjens, "Automatic estimation of the noise variance from the histogram of a magnetic resonance image," Phys Med Biol 52, 1335-1348 (2007).

25. M. Tanter, J. F. Aubry, J. Gerber, J. L. Thomas and M. Fink, "Optimal focusing by spatiotemporal inverse filter. I. Basic principles," J Acoust Soc Am 110, 37-47 (2001).

26. C. Mougenot, B. Quesson, B. D. de Senneville, P. L. de Oliveira, S. Sprinkhuizen, J. Palussiere, N. Grenier and C. T. Moonen, "Three-dimensional spatial and temporal temperature control with MR thermometry-guided focused ultrasound (MRgHIFU)," Magn Reson Med 61, 603-614 (2009).

27. F. J. Fry and J. E. Barger, "Acoustical properties of the human skull," J Acoust Soc Am 63, 1576-1590 (1978).

28. S. A. Sapareto and W. C. Dewey, "Thermal dose determination in cancer therapy," Int J Radiat Oncol Biol Phys 10, 787-800 (1984).

29. S. A. Sapareto, "Thermal isoeffect dose: addressing the problem of thermotolerance," Int J 
Hyperthermia 3, 297-305 (1987).

30. M. O. Köhler, C. Mougenot, B. Quesson, J. Enholm, B. Le Bail, C. Laurent, C. T. Moonen and G. J. Ehnholm, "Volumetric HIFU ablation under 3D guidance of rapid MRI thermometry," Med Phys 36, 3521-3535 (2009).

31. O. Seror, M. Lepetit-Coiffe, B. Le Bail, B. D. de Senneville, H. Trillaud, C. Moonen and B. Quesson, "Real time monitoring of radiofrequency ablation based on MR thermometry and thermal dose in the pig liver in vivo," Eur Radiol 18, 408-416 (2008). 


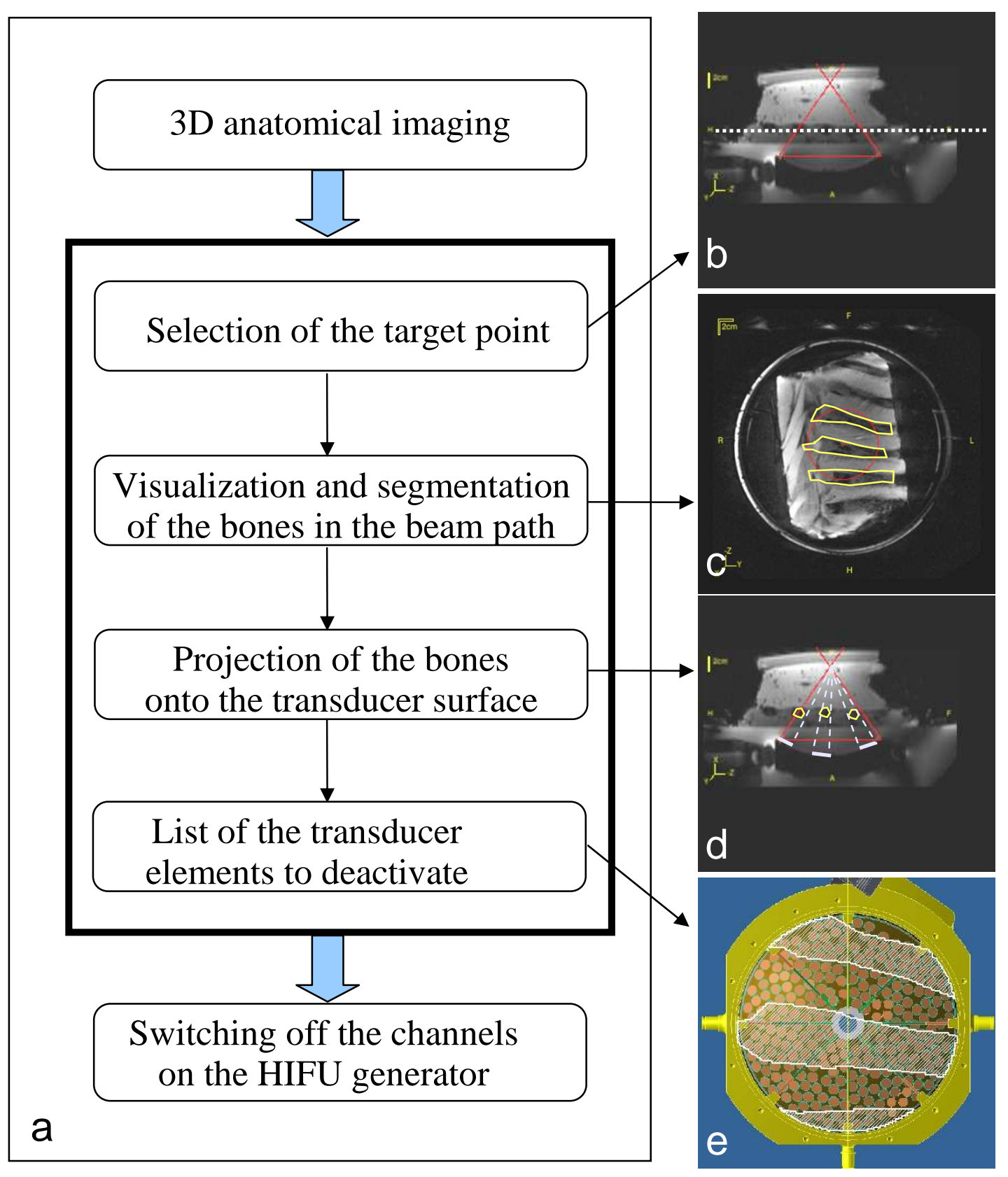

Figure 1 


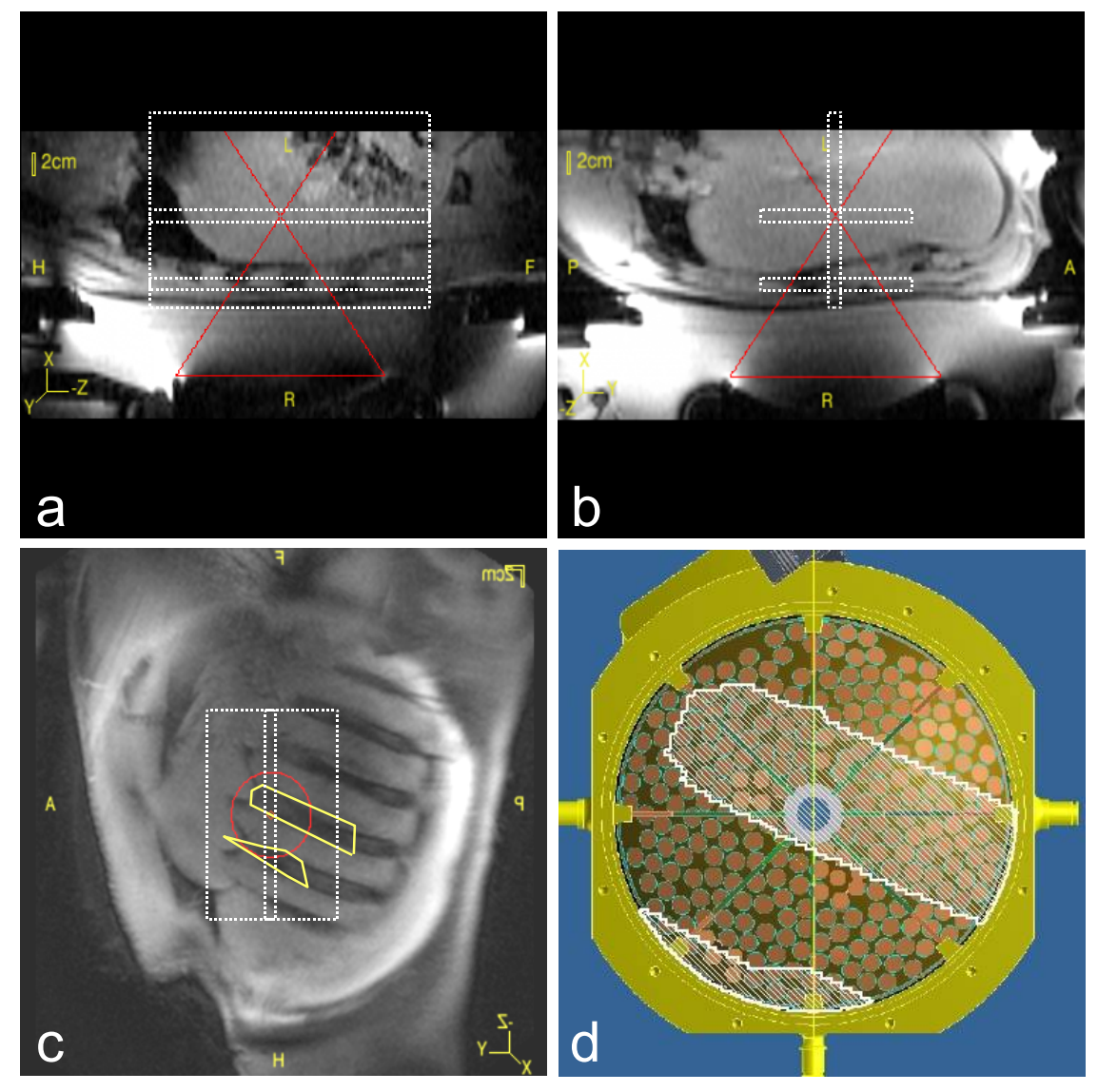

Figure 3 

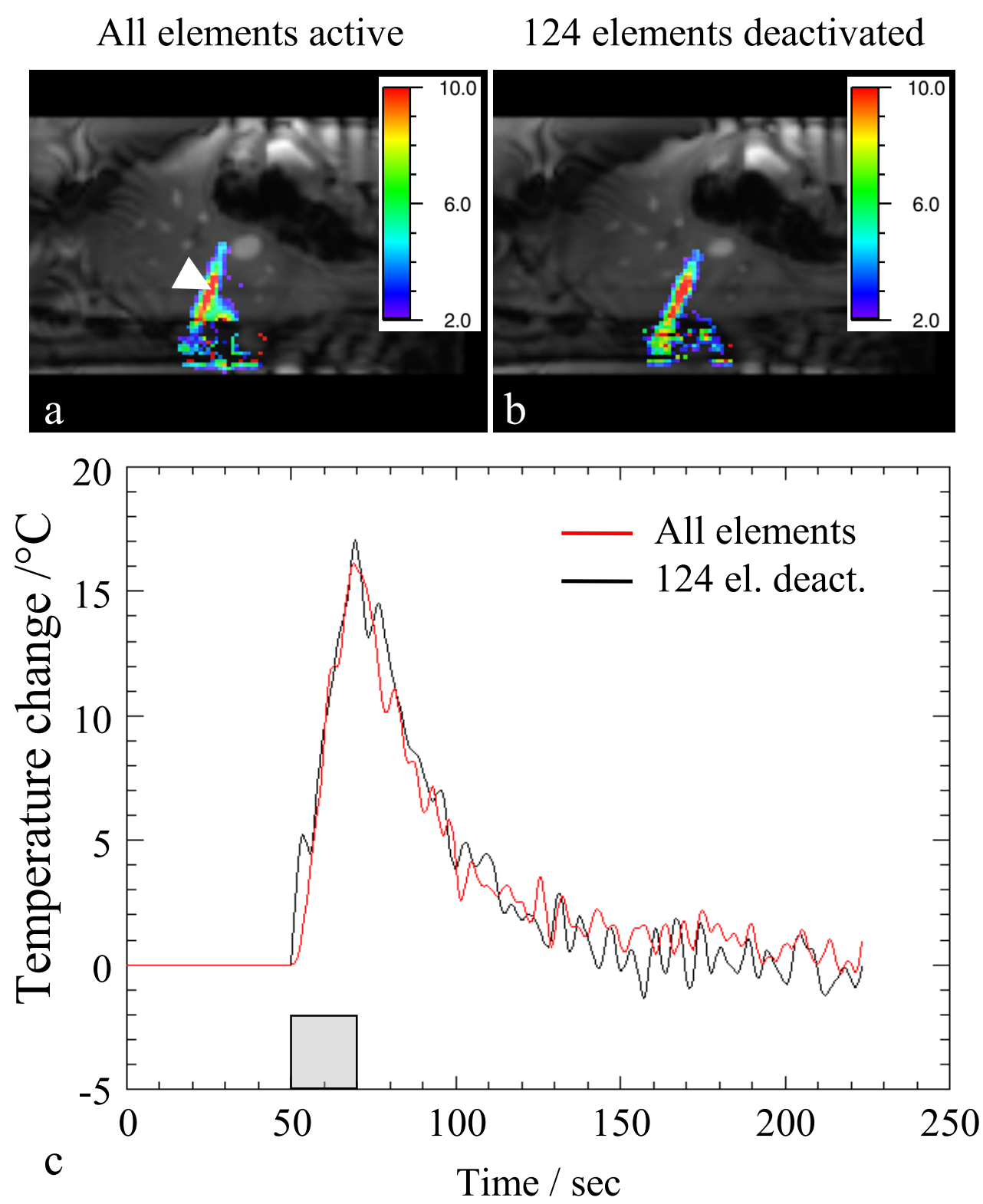

Figure 4 